15. J. Higgins, A. Conjusteau, G. Scoles, S. L. Bernasek J. Chem. Phys. 114, 5277 (2001)

16. M. J. Murphy, J. F. Skelly, A. Hodgson, B. Hammer J. Chem. Phys. 110, 6954 (1999).

17. A. C. Luntz, J. Chem. Phys. 113, 6901 (2000).

18. L. Diekhoner et al., J. Chem. Phys. 115, 9028 (2001)

19. D. C. Jacobs, J. Phys. Condens. Matter 7, 1023 (1995)

20. G. O. Sitz, Rep. Progr. Phys. 65, 1165 (2002).

21. P. R. McCabe, L. B. F. Juurlink, A. L. Utz, Rev. Sci. Instrum. 71, 42 (2000).

22. H. Hou et al., Science 284, 1647 (1999).

23. M. Gostein, G. O. Sitz, J. Chem. Phys. 106, 7378 (1997).
24. Details are available on Science Online.

25. K. Christmann, O. Schober, G. Ertl, M. Neumann, J. Chem. Phys. 60, 4528 (1974).

26. Q. Y. Yang, K. J. Maynard, A. D. Johnson, S. T. Ceyer, J. Chem. Phys. 102, 7734 (1995)

27. J. D. Beckerle, M. P. Casassa, R. R. Cavanagh, E. J. Heilweil, J. C. Stephenson, Phys. Rev. Lett. 64, 2090 (1990).

28. R. Milot, A. P. J. Jansen, Phys. Rev. B 61, 15657 (2000)

29. L. Halonen, S. L. Bernasek, D. J. Nesbitt, J. Chem. Phys. 115, 5611 (2001)

30. A. Bukoski, I. Harrison, J. Chem. Phys. 118, 9762 (2003).

\title{
The Structure of the First Coordination Shell in Liquid Water
}

\author{
Ph. Wernet, ${ }^{1,2}$ D. Nordlund, ${ }^{3}$ U. Bergmann, ${ }^{1}$ M. Cavalleri, ${ }^{3}$ \\ M. Odelius, ${ }^{3}$ H. Ogasawara, ${ }^{1,3}$ L. Å. Näslund, ${ }^{1,3}$ T. K. Hirsch, ${ }^{4}$ \\ L. Ojamäe, ${ }^{5}$ P. Glatzel, ${ }^{6}$ L. G. M. Pettersson, ${ }^{3}$ A. Nilsson ${ }^{1,3 *}$
}

\begin{abstract}
X-ray absorption spectroscopy and x-ray Raman scattering were used to probe the molecular arrangement in the first coordination shell of liquid water. The local structure is characterized by comparison with bulk and surface of ordinary hexagonal ice Ih and with calculated spectra. Most molecules in liquid water are in two hydrogen-bonded configurations with one strong donor and one strong acceptor hydrogen bond in contrast to the four hydrogen-bonded tetrahedral structure in ice. Upon heating from $25^{\circ} \mathrm{C}$ to $90^{\circ} \mathrm{C}, 5$ to $10 \%$ of the molecules change from tetrahedral environments to two hydrogen-bonded configurations. Our findings are consistent with neutron and $\mathrm{x}$-ray diffraction data, and combining the results sets a strong limit for possible local structure distributions in liquid water. Serious discrepancies with structures based on current molecular dynamics simulations are observed.
\end{abstract}

Experimental studies of the hydrogen-bonded network structure in water have mainly relied on neutron and $\mathrm{x}$-ray diffraction and infrared (IR) spectroscopies (1). Diffraction data from noncrystalline materials provide radial distribution functions (RDFs) (2-4) that do not provide angular correlations needed to uniquely assign local geometries in water (5-7). A more detailed atomistic picture has been derived theoretically by molecular dynamics (MD) simulations $(4,8)$ that are consistent with diffraction data. Structural information from IR spectroscopies generally relies on the correlation between the $\mathrm{O}-\mathrm{H}$ stretching frequency and hydrogen-bond (H-bond) length, which has been shown to be ambiguous for liquid water (9).

Here, we report an independent experimental investigation of local bonding configurations

${ }^{1}$ Stanford Synchrotron Radiation Laboratory, Post Office Box 20450, Stanford, CA 94309, USA. ${ }^{2}$ BESSY, Albert-Einstein-Strasse 15, D-12489 Berlin, Germany. ${ }^{3}$ FYSIKUM, Stockholm University, AlbaNova, S-10691 Stockholm, Sweden. ${ }^{4}$ Department of Physical Chemistry, Stockholm University, S-10691 Stockholm, Sweden. ${ }^{5}$ Department of Chemistry, Linköping University, S-58183 Linköping, Sweden. ${ }^{6}$ Department of Inorganic Chemistry and Catalysis, Debye Institute, Utrecht University, Sorbonnelaan 16, 3584 CA Utrecht, Netherlands.

*To whom correspondence should be addressed. Email: nilsson@slac.stanford.edu in the first coordination shell of liquid water by using the near-edge fine structure in $\mathrm{x}$-ray absorption spectroscopy (XAS), also denoted XANES and NEXAFS, where a core electron is excited into empty electronic states. The character of these states and, hence, the near-edge fine structure in XAS depends on the chemical environment, bond lengths, and bond angles (10). We also obtained the same spectral information by using nonresonant $\mathrm{x}$-ray Raman scattering (XRS) involving core excitations (11).

XAS and XRS at the oxygen K-edge (12) are sensitive to distortions of $\mathrm{H}$-bonds on the $\mathrm{H}$-sides (donor H-bonds) of the molecules in the condensed phases of water $(11,13,14)$. Because the time scale for excitation is much faster than the molecular (vibrational) motions in the liquid, these spectroscopies probe the electronic structure of a distribution of instantaneous configurations and thus allow decomposition in terms of specific H-bond situations (12). We analyzed the near-edge structures in the liquid water XA spectrum (the terminology "XA spectrum" is used for both XAS and XRS) with the aid of experimental model systems and calculated spectra. The XA spectra for water molecules in different H-bonding configurations are depicted in Fig. 1, where ice Ih bulk and surface spectra are compared with spectra of bulk liquid water at two temperatures. Bulk
31. R. D. Beck et al., Science 302, 98 (2003).

32. A. C. Luntz, Science 302, 70 (2003).

33. H. Mortensen, L. Diekhoner, A. Baurichter, A. C. Luntz, J. Chem. Phys. 116, 5781 (2002).

34. Supported by NSF (grant CHE-0111446) and Tufts University.

Supporting Online Material

www.sciencemag.org/cgi/content/full/304/5673/992/ DC1

Materials and Methods

2 February 2004; accepted 5 April 2004

ice Ih is tetrahedrally coordinated, but the exact $\mathrm{H}$-bonding environment at the ice Ih surface still raises questions $(15,16)$. However, there is consensus that a large fraction ( $50 \%$ or more) of the molecules in the first half bilayer of the ice Ih surface has one free O-H group, whereas the other is H-bonded to the second half bilayer. The liquid water XA spectrum closely resembles that for the ice surface, but it is very different from that of bulk ice. We interpret this finding, and our analysis demonstrates that the molecules in the liquid are not predominantly four-coordinated.

The spectra in Fig. 1 can be divided into three main regions: the pre-edge (around 535 $\mathrm{eV}$ ), the main edge (537 to $538 \mathrm{eV}$ ), and the post-edge (540 to $541 \mathrm{eV})$. The bulk ice spectrum (Fig. 1, curve a) is dominated by intensity in the post-edge region and shows a weak mainedge structure. Both the surface ice (Fig. 1, curve b) and liquid water (Fig. 1, curve d) spectra have a peak in the pre-edge region, a dominant main edge, and less intensity compared with bulk ice in the post-edge region. Termination of the ice surface with $\mathrm{NH}_{3}$ (Fig. 1, curve c) entails a coordination of the free $\mathrm{O}-\mathrm{H}$ groups and causes the pre-edge peak to vanish and the intensity to shift to the post-edge region. We assign intensities in the pre- and main-edge regions to water molecules with one uncoordinated $\mathrm{O}-\mathrm{H}$ group, whereas the intensity in the post-edge region is related to fully coordinated molecules. Remarkably, most molecules in bulk liquid water at room temperature exhibit a local coordination comparable to that at the ice surface, with one strong and one non-, or only weakly, H-bonded O-H group. The contribution to the spectrum from molecules with fourfold coordination similar to bulk ice is very small. Performing the measurements with $\mathrm{D}_{2} \mathrm{O}$ or $\mathrm{H}_{2} \mathrm{O}$ led to identical spectra within the experimental resolution, and thus tunneling contributions are not decisive.

Comparison of the XRS spectra of roomtemperature $\left(25^{\circ} \mathrm{C}\right)$ and hot water $\left(90^{\circ} \mathrm{C}\right)($ Fig. 1 , curve e) shows that heating increases intensities in the pre- and main-edge regions while decreasing that in the post-edge, but the changes are small compared with the changes observed between ice and the liquid. Figure 1, curve $\mathrm{f}$, shows the difference spectra of $25^{\circ} \mathrm{C}$ water minus ice (17) (solid curve) and $90^{\circ} \mathrm{C}$ minus $25^{\circ} \mathrm{C}$ water (circles with error bars). The latter has been multiplied by a factor of 10 to 


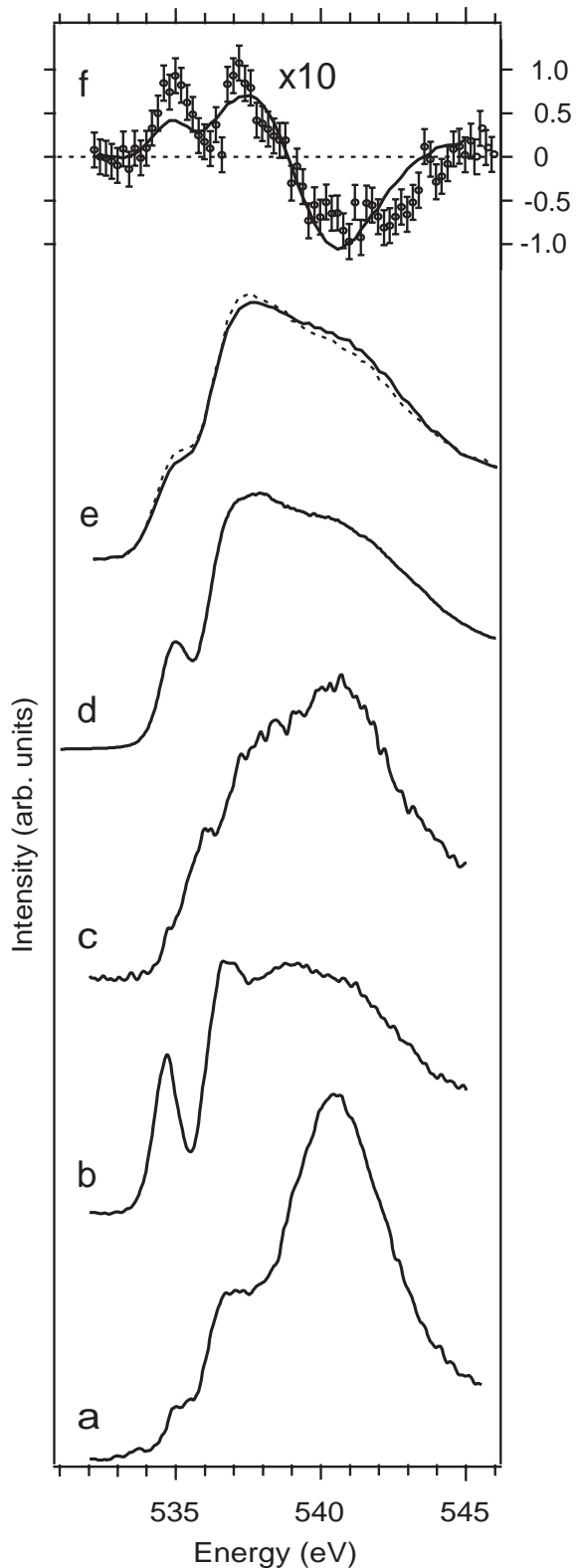

Fig. 1. (a) Bulk ice Ih (XAS secondary electron yield). (b) Ice Ih surface (topmost surface layer, XAS Auger electron yield). (c) $\mathrm{NH}_{3}-$ terminated first half bilayer of the ice lh surface (XAS Auger electron yield). (d) Liquid water at ambient conditions [XAS fluorescence yield, taken from (13) and additionally corrected for saturation effects]. (e) Bulk liquid water at $25^{\circ} \mathrm{C}$ (solid line) and $90^{\circ} \mathrm{C}$ (dashed line) (XRS, spectra normalized to the same area). (f) Difference spectra: $25^{\circ} \mathrm{C}$ water minus bulk ice (solid curve) and $90^{\circ} \mathrm{C}$ water minus $25^{\circ} \mathrm{C}$ water (circles with error bars). The latter difference spectrum has been multiplied by a factor of 10 . Note that XRS and XAS essentially give the same information, as indicated by the similarity of the room-temperature water spectra (e) and (d). Their slight differences are due to the poorer energy resolution of XRS. The temperature effects can best be studied with XRS because of the larger probing depth and the resulting easier sample preparation than for XAS. For details on sample preparation and probing depths, see $(11-13,15)$. match the intensities. The similarity of the curves, in particular the same isosbestic point at $538.8 \mathrm{eV}$, supports a two-component structure in terms of $\mathrm{O}-\mathrm{H}$ coordination: Configurations with one uncoordinated or weakly H-bonded $\mathrm{O}-\mathrm{H}$ group replace tetrahedral ones in the iceliquid phase transition, and heating liquid water causes similar types of changes, but of about one-tenth the magnitude. The contributions of the two different species at room temperature are $80 \%$ and $20 \%$ for species with only one strong H-bond and tetrahedrally coordinated, respectively; for $90^{\circ} \mathrm{C}$, we find $85 \%$ and $15 \%$ with uncertainties of \pm 15 to $\pm 20 \%$ in both cases (12).

We now compare this phenomenological finding with a detailed theoretical analysis based on density functional theory (DFT). The method used provides highly accurate results for both intensities and energy positions in $\mathrm{x}$ ray spectra of $\mathrm{H}$-bonded systems $(12,18)$. Here,

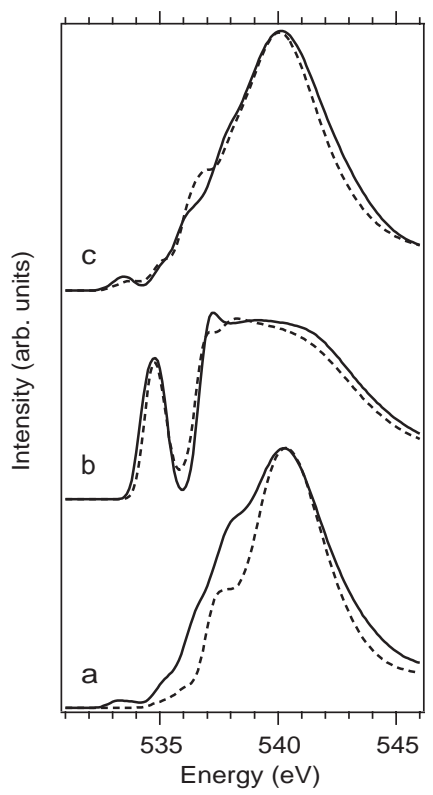

Fig. 2. Calculated spectra for the model systems bulk ice Ih. (a) Fully coordinated molecules. (b) Ice surface (molecules in the topmost surface layer with one free $\mathrm{O}-\mathrm{H}$ ). (c) $\mathrm{NH}_{3}$-terminated ice surface (molecules with an $\mathrm{NH}_{3}$-saturated $\mathrm{O}-\mathrm{H}$ ). Solid lines, small clusters; dashed lines, large clusters. Cluster sizes are 11 molecules for (a) to (c), solid lines; 44 molecules for (a), dashed line; and 27 molecules for (b) and (c), dashed lines. For the bulk ice cases, the calculated molecules are fully coordinated to their four nearest neighbors and surrounded by: (a, solid line) part of the second coordination shell ( 6 molecules) and (a, dashed line) the full second shell (12 molecules) and 27 further molecules. For the ice-surface cases, an appropriate number of molecules from the bulk ice clusters were removed [ 3 and 17 molecules for (b, solid line) and (b, dashed line), respectively] to generate configurations where the calculated molecule has one free $\mathrm{O}-\mathrm{H}$ as found on the ice surface $(12,15,16)$. To model the effect of $\mathrm{NH}_{3}$ termination, the same molecule was calculated after saturating the dangling $\mathrm{O}-\mathrm{H}$ by an $\mathrm{NH}_{3}$ molecule (lone pair electrons of the $\mathrm{N}$ oriented toward the free $\mathrm{O}-\mathrm{H}$ ). the calculations provide model spectra for various distributions of bond lengths and angles as expected to occur in the liquid. To allow for calculations of a large number of local configurations, we use a small model cluster of 11 molecules, where a central molecule is surrounded by the first coordination shell (4 molecules) plus part of the second shell (6 molecules saturating the dangling $\mathrm{O}-\mathrm{H}$ groups of the first shell). Spectra were calculated for the central molecule with systematically varied nearest neighbor H-bond environments.

First, the model cluster was tested by calculating the three known cases of bulk ice, ice surface, and $\mathrm{NH}_{3}$ terminated ice surface (Fig. 2). The calculated spectra based on clusters with 11 water molecules reproduce the general shapes and trends seen in the experimental spectra in Fig. 1: shift of intensity from post to pre and main edges when going from a fully coordinated molecule to one with a free $\mathrm{O}-\mathrm{H}$ group (compare Fig. 2, curves a and b) and shift of intensity back to the post-edge region when the free $\mathrm{O}-\mathrm{H}$ group is coordinated upon $\mathrm{NH}_{3}$ adsorption (compare Fig. 2, curves b and c).

These findings did not depend substantially on the cluster size: Calculated spectra for identical nearest neighbor $\mathrm{H}$-bond environments but now with a total of 44 (Fig. 2, curve a, dashed line) and 27 (Fig. 2, curves b and c, dashed lines) water molecules were similar to the spectra for small clusters. Furthermore, thermal fluctuations and/or vibrational motions did not affect the calculations considerably; for example, superposition of spectra with different intramolecular bonding distances was similar to that for the mean bonding configuration. The same holds for intermolecular vibrations (12).

To distinguish possible local configurations in the liquid, spectra were calculated for the central molecule in the 11-molecule cluster

Table 1. Relative amounts of local configurationsdouble-donor (DD), single-donor (SD), and nondonor (ND) configurations-in liquid water according to different $\mathrm{MD}$ simulations at $25^{\circ} \mathrm{C}$ and $90^{\circ} \mathrm{C}$. The values in the first column for each temperature follow from the experimental observations (EXP) in Fig. 1 (12) and from fitted spectra (FIT) such as the ones shown in Fig. 5. The errors are given by the uncertainties related to the experimental observations (Fig. 1) and the calculations (12).

\begin{tabular}{lcrrr}
\hline & \multicolumn{5}{c}{ Method } \\
\cline { 2 - 5 } Type & EXP + FIT & SPC & MCYL & CPMD \\
\hline & & $25^{\circ} \mathrm{C}$ & & \\
DD & $15_{-15}^{+25}$ & 70 & 50 & 79 \\
SD & $80 \pm 20$ & 27 & 41 & 20 \\
ND & $5 \pm 5$ & 3 & 9 & 1 \\
& & $90^{\circ} \mathrm{C}$ & & \\
DD & $10_{-10}^{+25}$ & 56 & 39 & 63 \\
SD & $85_{-20}^{+15}$ & 37 & 47 & 34 \\
ND & $5 \pm 5$ & 7 & 14 & 3 \\
\hline
\end{tabular}


with systematically varied nearest neighbor $\mathrm{H}$ bond distances and angles (Fig. 3A, curves a to h) (12). The spectrum for a configuration with tetrahedral coordination [linear H-bonds: all $\theta=0^{\circ}$ and $2.75 \AA$ for all O-O distances $(1,2)$, Fig. $3 \mathrm{~A}$, curve a] shows a maximum close to $540 \mathrm{eV}$, similar to Fig. 2, curve a. Donor Hbond distortions were introduced by varying the $\mathrm{O}-\mathrm{O}$ distance $r$ and the angle $\theta$ of the nearest neighbor (and the two attached molecules; see inset of Fig. 3A) on only one H-side (Fig. 3A, curves $\mathrm{b}$ to $\mathrm{e}$ ) and on both $\mathrm{H}$-sides (Fig. 3A, curves $f$ to $h$ ). Intermediate elongations smear out the maximum close to $540 \mathrm{eV}$ (Fig. 3A, curve b). Upon further distortion of one donor H-bond, a pre-edge peak emerges at $535 \mathrm{eV}$ and a mainedge structure develops at $537 \mathrm{eV}$, while postedge intensities decrease (Fig. 3A, curves c to e). Additional distortion of the second donor $\mathrm{H}$-bond (Fig. 3A, curves $\mathrm{f}$ and g) further reduces intensities above $540 \mathrm{eV}$ without considerable change in the pre- and main-edge regions. Note that varying $r$ at constant $\theta$ and varying $\theta$ at constant $r$ can yield similar spectra (e.g., Fig. 3A, curves c and e).

Spectra $\mathrm{c}$ to $\mathrm{e}$ and $\mathrm{g}$ to $\mathrm{h}$ in Fig. 3A show that donor $\mathrm{H}$-bond distortions of a certain degree manifest as a distinct pre-edge peak and an intense main edge in the oxygen K-edge XA spectrum of water. The analogy of these preand main-edge features to the ones observed for the ice-surface molecules with one broken do-

nor H-bond (Figs. 1 and 2) suggests denoting the corresponding distorted H-bonds as "broken." More specifically, based on spectral calculations of a large number of different configurations in the liquid, we define the cutoff for $\mathrm{H}$-bonding in terms of $\mathrm{O}-\mathrm{O}$ distances as the boundary between zones A and B (black line in Fig. 3B) (12). For linear H-bonds, we find the same cutoff as conventionally used in the analysis of MD simulations (19). For bent H-bonds, we use a smaller cutoff because of the occurrence of pre- and main-edge intensities for large angular distortions at standard $\mathrm{O}-\mathrm{O}$ distances (Fig. 3A, curve e). This difference is not important, as will be addressed in detail below.

Zones A and B in Fig. 3B can be used to classify local water configurations: The environment in bulk ice, e.g., is an AA configuration, with both nearest neighbor H-bond acceptor molecules within zone $\mathrm{A}$, whereas that at the ice surface is an $\mathrm{AB}$ configuration. We define three groups of configurations in the liquid: Double-donor (DD) configurations with two intact donor H-bonds are characterized by XA spectra without pre-edge peak (Fig. 3A, curves a, b, and f); single-donor (SD) configurations with one intact and one broken donor H-bond exhibit a distinct pre-edge peak and an intense main edge (Fig. 3A, curves c, d, e, and $\mathrm{g}$ ); and the nondonor (ND) configuration with two broken donor H-bonds gives two distinct peaks (Fig. 3A, curve h) similar to the

A

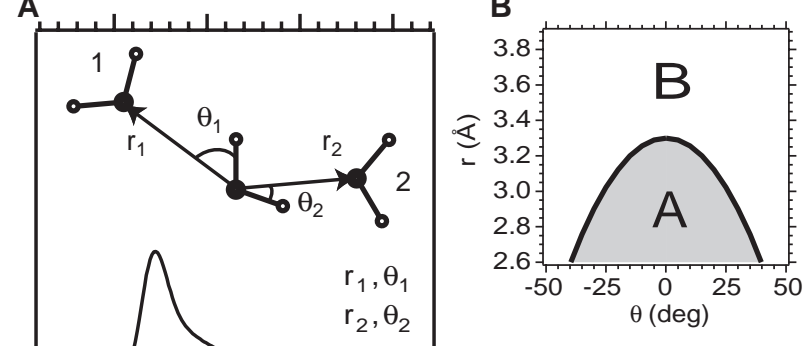

Fig. 3. (A) Calculated spectra for a cluster of 11 water molecules with $\mathrm{O}-\mathrm{O}$ distances $r_{\mathrm{i}}$ (in $\AA$ ) and angles $\theta_{i}$ (in degrees) of the nearest neighbors $i=1,2$ on the $\mathrm{H}$-sides (nearest neighbor $\mathrm{H}$-bond acceptor molecules) systematically varied as given for each spectrum. Starting from (a) a tetrahedral structure with all four nearest neighbor $\mathrm{O}-\mathrm{O}$ distances at $2.75 \AA$ and linear $\mathrm{H}$-bonds, for (b, c) only $r_{1}$, for (d) $r_{1}$ and $\theta_{1}$, and for (e) only $\theta_{1}$ are varied to introduce $\mathrm{H}$-bond distortions on one $\mathrm{H}$-side. For (f) $\theta_{1}$ and $r_{2}$ and for $(\mathrm{g}, \mathrm{h}) r_{1}$ and $r_{2}$ are varied to introduce $\mathrm{H}$-bond distortions on both $\mathrm{H}$-sides. Compared with the cluster calculation for ice Ih in Fig. 2, curve a, solid line, relative orientations of the molecules have been changed arbitrarily to better account for the less ordered local configurations in the liquid. In addition, the calculated oscillator strengths are convoluted with a slightly larger width to account for the larger vibrational motion of the molecules in the liquid. This explains the differences between (a) and Fig. 2, curve a, solid line. (B) The systematic spectral changes allow for a definition of zones $A$ and $B$ to denote the positions of the two nearest neighbor $\mathrm{H}$-bond acceptor molecules. Spectra show similar features for positions within one zone [compare, e.g., (a) and (b) or (c) to (e) and as verified by many further calculations], and the cases shown here can be considered to be representative. The boundary between the zones (black line) is used as a cutoff for $\mathrm{H}$-bonding (12) as based on the occurrence of strong pre- and main-edge features (similar to the experimental ice surface spectrum) when the nearest neighbor is outside zone $A$ beyond the black line (e.g., c to e). spectra of gas-phase water and the species at the surface of liquid water (20).

The pre-edge intensities for the SD configurations are due to excitations to unoccupied antibonding orbitals that are localized along the internal O-H bond (Fig. 4). The localization is caused by breaking one donor H-bond while keeping the other intact, which entails s-p-rehybridization in the orbitals close to $535 \mathrm{eV}$ (14). The resulting increase of p-character translates to an intensity increase in the pre-edge region of the XA spectra through the dipole selection rule (12). The orbital that contributes to the pre-edge intensity closely matches zone A, as shown in Fig. 4. This comparison gives our H-bond criterion its physical basis and supports our interpretation; the presence of another molecule inside zone A changes this orbital and thereby the corresponding spectral feature, which also explains the sensitivity of XAS (XRS) to H-bond distortions on the $\mathrm{H}$-side.

The occurrence of the pre-edge feature, furthermore, can be directly correlated with the energetics in the H-bond interaction. For the model clusters considered here, we find that distortions leading to enhanced intensity in the pre-edge correspond to one donor H-bond losing about 40 to $70 \%$ of the individual bond energy of bulk ice (12). The SD species that dominate the liquid thus have one strong and one weak or broken donating H-bond. Consistent with commonly used definitions of $\mathrm{H}$-bonds in liquid water (and for simplicity), we use the term "broken" for the distorted donor H-bond of an SD configuration.

In Fig. 3A, only the calculated spectra of SD species in curves $\mathrm{c}$ to $\mathrm{e}$ and $\mathrm{g}$ exhibit all features observed in the experimental liquid water spec-

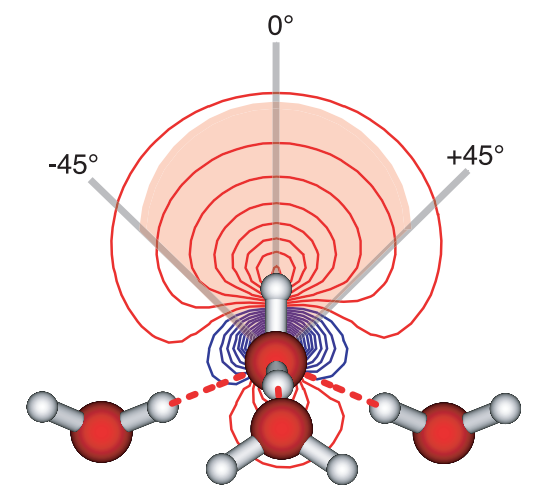

Fig. 4. Connecting electronic and geometric structures. The contour plot of a typical unoccupied orbital giving rise to the pre-edge intensity in the XA spectrum of a water molecule with a broken donor $\mathrm{H}$-bond (solid line contour plot) is compared with our cutoff criterion for $\mathrm{H}$-bonding (black line in Fig. 3B) (12). Broken donor $\mathrm{H}$-bonds are characterized by nearest neighbor O's outside the shaded area (12). The orbital contour has been calculated for the central molecule in a cluster with 11 molecules (the corresponding XA spectrum is shown in Fig. 2, curve b, solid line) where the $\mathrm{H}$-bond on one $\mathrm{H}$-side is broken (SD configuration). Only 4 of the 11 molecules are displayed. 
trum: a pre-edge peak close to $535 \mathrm{eV}$ and a strong main edge close to $537 \mathrm{eV}$. This result suggests, consistent with our phenomenological findings, that asymmetric SD species with one strong and one broken donor H-bond dominate the liquid water structure. In a more detailed analysis, our experimental data were fitted with sums of calculated spectra. The results are summarized in Table 1. Different combinations of computed spectra corresponding to different choices of bond lengths and angles within each class give similar spectra. All yield a dominant contribution of SD species both at $25^{\circ} \mathrm{C}$ and $90^{\circ} \mathrm{C}$. We note the close agreement with the previously described analysis based on model experimental spectra (12). For $90^{\circ} \mathrm{C}$ we find the best fits for a decreased (increased) amount of DD (SD) species as compared with room temperature, indicating the breaking of H-bonds with increasing temperature along with the decrease of fully coordinated molecules (21). In particular, it comes as no surprise that bulk-ice-like configurations are virtually absent in water close to the boiling point. Consistent with the discussion of Fig. 1, we find that DD configurations are replaced by SD configurations both when heating the liquid and when melting ice into liquid water. Computed spectra indicate that XAS is not sensitive to the breaking of H-bonds on the O-side (acceptor H-bond) (13). However, symmetry arguments imply that, with a dominant amount of molecules in SD configurations, most molecules must in addition have a broken acceptor $\mathrm{H}$-bond. This entails that, within the H-bond definition given here [Fig. 3 and (12)], each molecule has on average $2.2 \pm 0.5$ (2.1 \pm $0.5) \mathrm{H}$-bonds at $25^{\circ} \mathrm{C}\left(90^{\circ} \mathrm{C}\right)$.

As mentioned above, XAS cannot distinguish between SD configurations with a broken bond due to elongation (Fig. 3A, curve c) or to strong bending (Fig. 3A, curve e). However, these configurations exhibit different nearest neighbor $\mathrm{O}-\mathrm{O}$ and $\mathrm{O}-\mathrm{H}$ distances. We compare our results with $\mathrm{O}-\mathrm{O}$ and $\mathrm{O}-\mathrm{H}$ RDFs from neutron diffraction data taken at ambient conditions $(2,22)$. Two XA spectra generated with different distributions of H-bond lengths and angles, but both with the same fraction of DD, SD, and ND species, are shown in Fig. 5A, curves $a$ and $b$. Spectra of this type have been used to estimate the amounts of the different species (Table 1) that are consistent with the measured XA spectrum.

The O-O (Fig. 5B) and O-H (Fig. 5C) RDFs are shown for the two model distributions corresponding to the XA spectra in Fig. 5A, curves $\mathrm{a}$ and $\mathrm{b}$ (12). The 11-molecule model configurations that can adequately describe the local structure probed by XAS are obviously not sufficient for a full description of RDFs beyond the first coordination shell $[<3.4$ to $3.5 \AA$ in the O-O RDF $(2,4)$ and $<2.5$ to $2.6 \AA$ in the O-H RDF (2)]. Molecules from the second shell are not included in the RDFs of models a to $\mathrm{c}$ in Fig. 5 and hence we should not expect agreement for larger distances. The "a" model has a larger portion of broken $\mathrm{H}$-bonds as a result of
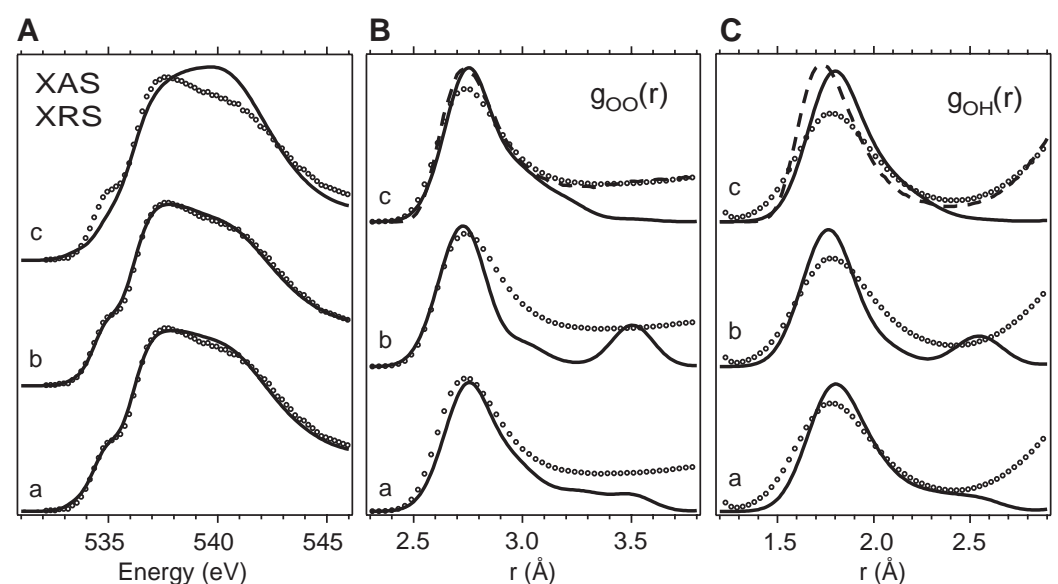

Fig. 5. Calculated XA spectra and RDFs (solid lines) for three models-(a), (b), and (c)-are compared with (A) experimental XA spectra and RDFs (B and C) at $25^{\circ} \mathrm{C}$. Spectra and RDFs have been generated simultaneously for the central molecule in clusters consisting of 11 molecules, for a total of 14 clusters (12). A sampling of the possible DD, SD, and ND configurations for the calculated molecule in the liquid is guaranteed by using a variety of nearest neighbor $\mathrm{O}-\mathrm{O}$ distances and $\mathrm{H}$-bond angles. The RDFs were calculated for the first coordination shell only. Experimental RDFs [open circles in (B) and (C)] were derived from neutron diffraction (2). The calculated XA spectra were broadened with a $1 \mathrm{eV}$ Gaussian for better comparison with experimental XRS data [open circles in (A)] taken from Fig. 1, curve e. Models (a) and (b) use a balance of DD:SD:ND = 10:85:5. Model a (b) has more (less) angularly distorted H-bonds and less (more) elongated ones. Model (c) (solid line) represents the SPC simulation (Table 1) with a balance DD:SD:ND = 70:27:3, as determined with our criterion for $\mathrm{H}$-bonding (12). For comparison, the dashed lines in $\mathrm{B}$ and $\mathrm{C}$, curve $\mathrm{c}$, show the O-O and O-H RDFs calculated from the full SPC simulation. There is good agreement up to $3.2 \AA(\mathrm{O}-\mathrm{O})$ and $2.4 \AA(\mathrm{O}-\mathrm{H})$, validating our approach to characterize the first shell in the liquid. Only model (a) or similar balances with a dominant fraction of SD species (Table 1) give good fits when simultaneously considering our experimental XRS data and the RDF curves. bending, whereas the " $b$ " model has more elongated bonds. Both models reproduce the experimental XA spectrum, but model a clearly appears to provide a better agreement with the RDFs within the first coordination shell. Model b lacks structural contributions in the region 2.9 to 3.3 (1.9 to 2.3$) \AA$ and overemphasizes the contributions around 3.5 and $2.5 \AA$. It is thus inconsistent with water density, as illustrated by the poor agreement with the diffraction data. Combining XAS and diffraction results therefore suggests that the H-bonds are predominantly broken by bending rather than by elongation.

Finally, our results can be compared with two classical MD simulations using the flexible SPC (23) and MCYL (24) pair potential energy models and to an ab initio Car-Parrinello MD (CPMD) simulation (25) [for details of the simulations, see (12)]. The sampling of the distribution of structures is similar to that in the XAS/XRS experiment, i.e., the instantaneous geometries at a sequence of time steps are analyzed. Using our criterion for H-bonding (12), we have determined the amounts of DD, $\mathrm{SD}$, and ND configurations (Table 1). The MCYL potential yields the largest amount of $\mathrm{SD}$, but all of the simulations deviate substantially from experimental results. Note that with our spectroscopically determined electronic structure criterion, SPC (CPMD) at $25^{\circ} \mathrm{C}$ still gives an average of 3.3 (3.6) H-bonds per molecule, similar to earlier theoretical studies reporting $3.5 \mathrm{H}$-bonds per molecule (19). For example, with the numbers in Table 1 for SPC at $25^{\circ} \mathrm{C}$, we determine that $(0.7 \times 4)+(0.27 \times$ $2)+(0.03 \times 0)=3.34$ H-bonds per molecule. An XA spectrum generated based on the distribution of species obtained from our SPC MD simulation at $25^{\circ} \mathrm{C}$ is shown in Fig. $5 \mathrm{~A}$, curve c. Clearly the agreement with the experimental spectrum is very poor: no pre-edge intensity (SD species) and too much intensity around 540 $\mathrm{eV}$ (fully coordinated DD species). The corresponding $\mathrm{O}-\mathrm{O}(\mathrm{O}-\mathrm{H}) \mathrm{RDFs}(12)$ are depicted in Fig. 5B (C), curve c, as solid lines. We note that the SPC simulation, in comparison with experimental results, exaggerates contributions at $r$ values characteristic for tetrahedral/near tetrahedral configurations (in the maxima) in both the O-O and O-H RDFs. However, the O-O (O-H) RDFs from SPC and our model a are rather similar at 2.9 to 3.3 (1.9 to 2.3 ) $\AA$. The poor agreement with the experimental XA spectrum is hence attributed to too few species with large H-bond angles (SD species) in the SPC simulation. Similarly, the other two MD simulations shown in Table 1 cannot create an XA spectrum that fits the data. The MD simulations are, however, consistent with our results in predicting the decrease (increase) of DD (SD) species with increasing temperature.

The large amount of SD species can also explain femtosecond-IR studies indicating two different $\mathrm{O}-\mathrm{H}$ groups in liquid water: one "strongly" and one "weakly" H-bonded (26). 
The orientational relaxation dynamics were shown to be directly connected with the strength of the two H-bond groups, where the weak H-bonds relax much faster than the strong H-bonds. According to our results, the number of strong $\mathrm{H}$-bonds in the liquid is substantially smaller than expected, which may seem in contradiction with the small heat of melting compared with the heat of sublimation for ice. However, quantum chemical calculations have shown that each bond in the proposed SD configurations is stronger than the average bond in four-fold coordination because of anticooperativity effects $(27,28)$. Thus, the large number of weakened/broken $\mathrm{H}$-bonds in the liquid leads to only a small change in energy. A recently developed quantum chemical model $(1,27)$ that proposes the predominance in the liquid phase of two hydrogen-bonded water molecules in ring conformations is consistent with our results. Water is a dynamic liquid where $\mathrm{H}$ bonds are continuously broken and reformed (29). The present result that water, on the probed subfemtosecond time scale, consists mainly of structures with two strong H-bonds, one donating and one accepting, nonetheless implies that most molecules are arranged in strongly $\mathrm{H}$-bonded chains or rings embedded in a disordered cluster network connected mainly by weak H-bonds.

\section{References and Notes}

1. R. Ludwig, Angew. Chem. Int. Ed. Engl. 40, 1808 (2001).

2. A. K. Soper, Chem. Phys. 258, 121 (2000).

3. A. H. Narten, H. A. Levy, Science 165, 447 (1969).

4. T. Head-Gordon, G. Hura, Chem. Rev. 102, 2651 (2002).

5. P. G. Kusalik, I. M. Svishchev, Science 265, 1219 (1994).

6. A. K. Soper, J. Chem. Phys. 101, 6888 (1994).

7. A. A. Chialvo, P. T. Cummings, J. Phys. Chem. 100 1309 (1996)

8. F. H. Stillinger, Science 209, 451 (1980)

9. J. Stenger, D. Madsen, P. Hamm, E. T. J. Nibbering, T. Elsaesser, Phys. Rev. Lett. 87, 027401 (2001).

10. J. Stöhr, NEXAFS Spectroscopy (Springer-Verlag, Berlin, 1992).

11. U. Bergmann et al., Phys. Rev. B 66, 092107 (2002)

12. Details of the materials and methods, and supporting analysis of the experimental data, are available at Science Online.

13. S. Myneni et al., J. Phys. Condens. Matter 14, L213 (2002).

14. M. Cavalleri, H. Ogasawara, L. G. M. Pettersson, A Nilsson, Chem. Phys. Lett. 364, 363 (2002).

15. D. Nordlund et al., unpublished data.

16. A. Glebov, A. P. Graham, A. Menzel, J. P. Toennies, P. Senet, J. Chem. Phys. 112, 11011 (2000).

17. The ice spectrum (Fig. 1, curve a) has been broadened to the same instrumental resolution as for XRS and normalized to the same area before subtraction.

18. M. Nyberg, M. Odelius, A. Nilsson, L. G. M. Pettersson, J. Chem. Phys. 119, 12577 (2003).

19. A. Luzar, D. Chandler, Phys. Rev. Lett. 76, 928 (1996)

20. K. R. Wilson et al., J. Phys. Condens. Matter 14, L221 (2002).

21. The sharper main edge for hot water (537 eV, Fig. 1, curve e, dashed line) is accounted for by a different balance inside the group of SD species, where more of the $\mathrm{H}$-bond acceptor molecules have distances closer to the $A B$ boundary, i.e., have a larger $r_{2}$ and/or $\theta_{2}$ but still remain in zone A (more Fig. 3A, curve g, and less curve c, e.g.). Accordingly, the intensity decrease above the isosbestic point with temperature can be mainly assigned to the loss of DD configurations with tetrahedral and near tetrahedral environments by 5 to $10 \%$ and an increase of SD configura- tions with $\mathrm{H}$-bond acceptor molecules being closer to the $A B$ boundary.

22. The neutron O-O RDF in (2) is very similar to the most recently derived O-O RDF from $x$-ray diffraction in (4).

23. K. Toukan, A. Rahman, Phys. Rev. B 31, 2643 (1985)

24. G. C. Lie, E. Clementi, Phys. Rev. A. 33, 2679 (1986)

25. P. L. Silvestrelli, M. Parrinello, J. Chem. Phys. 111 3572 (1999).

26. S. Woutersen, U. Emmerichs, H. J. Bakker, Science 278, 658 (1997).

27. F. Weinhold, J. Chem. Phys. 109, 373 (1998)

28. L. Ojamäe, K. Hermansson, J. Phys. Chem. 98, 4271 (1994).

29. C. J. Fecko, J. D. Eaves, J. J. Loparo, A. Tokmakoff, P. L. Geissler, Science 301, 1698 (2003).

30. Supported by the Swedish Foundation for Strategic Research, Swedish Natural Science Research Council, and U.S. National Science Foundation grant CHE-0089215. Generous grants of computer time at the Swedish National Supercomputer Center and the Center for Parallel Computing are gratefully acknowledged. Portions of this research were carried out at the Stanford Synchrotron Radiation Laboratory, a national user facility operated by Stanford University on behalf of the U.S. Department of Energy Office of Basic Energy Sciences. Use of the Advanced Photon Source (APS) was supported by the U.S. Depart- ment of Energy, Basic Energy Sciences, Office of Science, under contract No. W-31-109-ENG-38. Biophysics Collaborative Access Team (BioCAT) is a National Institutes of Health-supported Research Center RR-08630. The Advanced Light Source (ALS) is supported by the Director, Office of Science, Office of Basic Energy Sciences, Materials Sciences Division, of the U.S. Department of Energy under Contract No. DE-AC03-76SF00098 at Lawrence Berkeley National Laboratory. Assistance by the APS, ALS, and the Swedish national laboratory MAX-lab staff is gratefully acknowledged. We thank J. B. Hastings for his valuable comments and discussions and S. P. Cramer for making the XRS spectrometer available.

\section{Supporting Online Material}

www.sciencemag.org/cgi/content/full/1096205/DC1 Materials and Methods

SOM Text

Figs. S1 to S11

Tables S1 and S2

References and Notes

29 January 2004; accepted 25 March 2004

Published online 1 April 2004;

10.1126/science. 1096205

Include this information when citing this paper.

\title{
Asphalt Volcanism and Chemosynthetic Life in the Campeche Knolls, Gulf of Mexico
}

\author{
I. R. MacDonald, ${ }^{1 *}$ G. Bohrmann, ${ }^{2}$ E. Escobar, ${ }^{3}$ F. Abegg, ${ }^{2}$ \\ P. Blanchon, ${ }^{4}$ V. Blinova, ${ }^{2}$ W. Brückmann, ${ }^{5}$ M. Drews, ${ }^{5}$ \\ A. Eisenhauer, ${ }^{5}$ X. Han, ${ }^{6}$ K. Heeschen, ${ }^{2}$ F. Meier, ${ }^{2}$ C. Mortera, ${ }^{7}$ \\ T. Naehr, ${ }^{1}$ B. Orcutt, ${ }^{8}$ B. Bernard, ${ }^{9}$ J. Brooks, ${ }^{9}$ M. de Faragó ${ }^{10}$
}

In the Campeche Knolls, in the southern Gulf of Mexico, lava-like flows of solidified asphalt cover more than 1 square kilometer of the rim of a dissected salt dome at a depth of $\mathbf{3 0 0 0}$ meters below sea level. Chemosynthetic tubeworms and bivalves colonize the sea floor near the asphalt, which chilled and contracted after discharge. The site also includes oil seeps, gas hydrate deposits, locally anoxic sediments, and slabs of authigenic carbonate. Asphalt volcanism creates a habitat for chemosynthetic life that may be widespread at great depth in the Gulf of Mexico.

Salt tectonism in the Gulf of Mexico hydrocarbon province controls the development of reservoirs and faults that allow oil

${ }^{1}$ Physical and Life Sciences Department, Texas A \& M University-Corpus Christi, 6300 Ocean Drive, Corpus Christi, TX 78412, USA. ²Fachbereich 5 Geowissenschaften, University Bremen, D-28334 Bremen, Germany. ${ }^{3}$ Universidad Nacional Autonoma de Mexico, Instituto de Ciencias del Mar y Limnologìa, Apartado Postal 70-305, Mexico 045510, D.F. Mexico. ${ }^{4}$ Instituto de Ciencias del Mar y Limnologìa, Apartado Postal 1152, Cancún, D.F. Mexico. 5 IFM-GEOMAR, Leibniz-Institut für Meereswissenschaften, D-24148 Kiel, Germany. ${ }^{6}$ Second Institute of Oceanography, State Oceanic Administration, Zhejiang 310012, China. ${ }^{7}$ Universidad Nacional Autonoma de Mexico, Instituto de Geofisica, Mexico, 04510, D.F. Mexico. ${ }^{8}$ University of Georgia, 220 Marine Sciences Building, Athens, GA 30602, USA. 9TDI-Brooks International, Inc. 1902 Pinion, College Station, TX 77845, USA. ${ }^{10}$ Aurensis, SA, San Francisco de Sales 38, 28003, Madrid, Spain.

*To whom correspondence should be addressed. Email: imacdonald@falcon.tamucc.edu and gas to escape at the sea floor (1). More than 30 years ago, investigators studying the Gulf's abyssal petroleum system (2) photographed an asphalt deposit (3) among salt domes in the southern Gulf of Mexico. During exploration of the Campeche Knolls, about $200 \mathrm{~km}$ south of the photographed site (Fig. 1, A and C), we have now found numerous, deeply dissected salt domes with extensive slumps and mass wasting at depths of $3000 \mathrm{~m}$ or greater. Massive, lava-like flow fields of solidified asphalt, evidently discharged at temperatures higher than the ambient bottom water $\left(4^{\circ} \mathrm{C}\right)$, have been colonized by an abundant chemosynthetic fauna.

The Campeche Knolls are salt diapirs rising from an evaporite deposit that underlies the entire slope region (4) and hosts the Campeche offshore oil fields (5). Numerous reservoir and seal facies have also been at- 